\title{
The value of a single isolated CTG trace deceleration
}

\author{
Juan Piazze ${ }^{1 *}$, Francesco Recchia ${ }^{2}$, Costantino Testani ${ }^{3}$ and Silvio Rea ${ }^{4}$ \\ ${ }^{1}$ ASL Frosinone (Casa della Salute di Ceprano-Ospedale SS Trinità di Sora), Fondazione Carlo Ferri Monterotondo, Rome \\ ${ }^{2}$ Ospedale Civile di Avezzano, Fondazione "Carlo Ferri", Monterotondo, Rome \\ ${ }^{3}$ Ospedale SS Trinità di Sora, Frosinone \\ ${ }^{4}$ Chirurgia Oncologica, Università degli Studi de L'Aquila, Dpt DISCAB, Fondazione “Carlo Ferri”, Monterotondo, Roma
}

\begin{abstract}
We report one case regarding the validity of a single isolated FHR deceleration in one pregnant woman a term affected by hypertension in pregnancy, whose follow-up was uneventful until the day before a cesarean section was performed.
\end{abstract}

\section{Introduction}

Fetal heart rate (FHR) monitoring has been used antepartum as a screening test to establish fetal health. Cardiotocography (CTG) is a method of determining fetal well-being that may be used on a daily basis, involving the recording of FHR and uterine contractility over a period of time, usually 20-40 minutes. CTG recording should not be used to assess primarily only FHR variability, as it usually occurs, but should be kept in mind that, when satisfactory, it ensures clinicians that cerebral control of FHR variability through the autonomic nervous system is intact, reflecting absence of fetal cerebral acidosis [1]. An abnormal FHR pattern does not always is related to fetal hypoxia. Fetal heart rate deceleration patterns take different forms used for clinical diagnosis (early, variable and late deceleration) [2]. Late variant indicates uteroplacental insufficiency. Compared with these forms of heartbeat decelerations, a bradycardic episode without associated uterine activity is defined as spontaneous deceleration [3].

In this case we would like to underline the clinical meaning of spontaneous FHR decelerations, in order to decide if the most suitable management for timing of delivery.

\section{Case report}

A 32 year old G1 P1 woman presented to our Institution for evaluation at $38+4$ wks gestation.

She was affected by hypertension under specific therapy that kept blood pressure parameters under control, ad demonstrated in our Department, where she arrived for a regular CTG trace as required in hypertension cases. A color Doppler velocimetry was performed two weeks before showing, as stated in the report taken under vision, a normal ultrasound examination.

After a 40 minutes CTG trace, one single deceleration was observed within the first fetal quiescence phase of the whole trace, with FHR decaying from 140 to 120 , keeping a slight variability in deep, and returning to a baseline variability between $5-10 \mathrm{bpm}$ (Figure 1). After about 20 minutes CTG rhythm increased variability (from 10-25 bpm), with no other deceleration present. No signs of uterine activity were observed in all the CTG trace. With these findings, the CTG trace was considered not reassuring and the patient was scheduled for e new CTG trace the day after.
The day after a 40 minutes CTG trace was performed presenting a rhythmic uterine contraction, FHR at $140 \mathrm{bpm}$, a variability from $0-5$ bpm and one mild early deceleration (Figure 2).

An ultrasound scan was immediately performed showing oligohydramnios (AFI $7.1 \mathrm{~cm}$ ), with a normal fetal-maternal color Doppler.

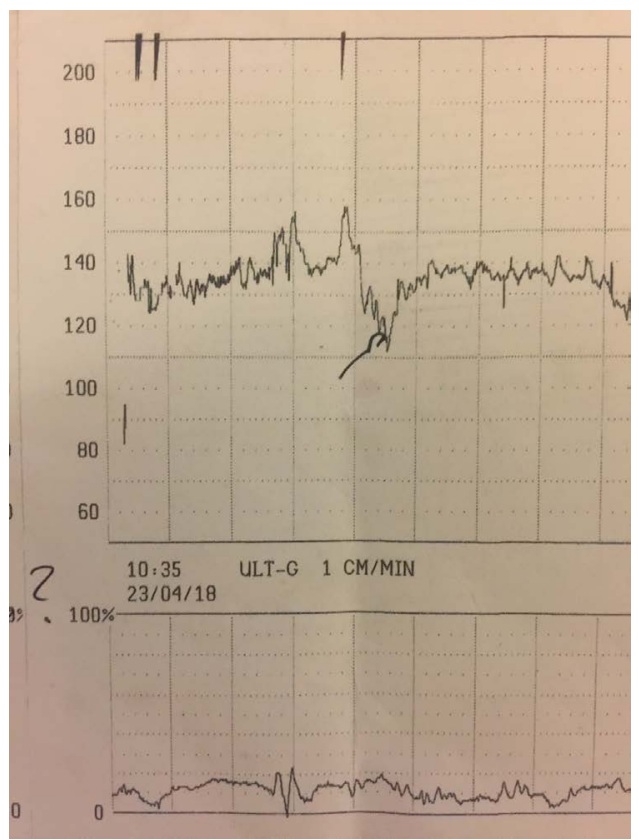

Figure 1. Isolated spontaneous FHR trace deceleration

${ }^{\star}$ Correspondence to: Juan Piazze, ASL Frosinone (Casa della Salute di CepranoOspedale SS Trinità di Sora), Fondazione Carlo Ferri Monterotondo, Rome, E-mail: cloudpia@me.com

Key words: isolated deceleration, ultrasound, fetal well-being

Received: April 19, 2018; Accepted: April 25, 2018; Published: April 30, 2018 


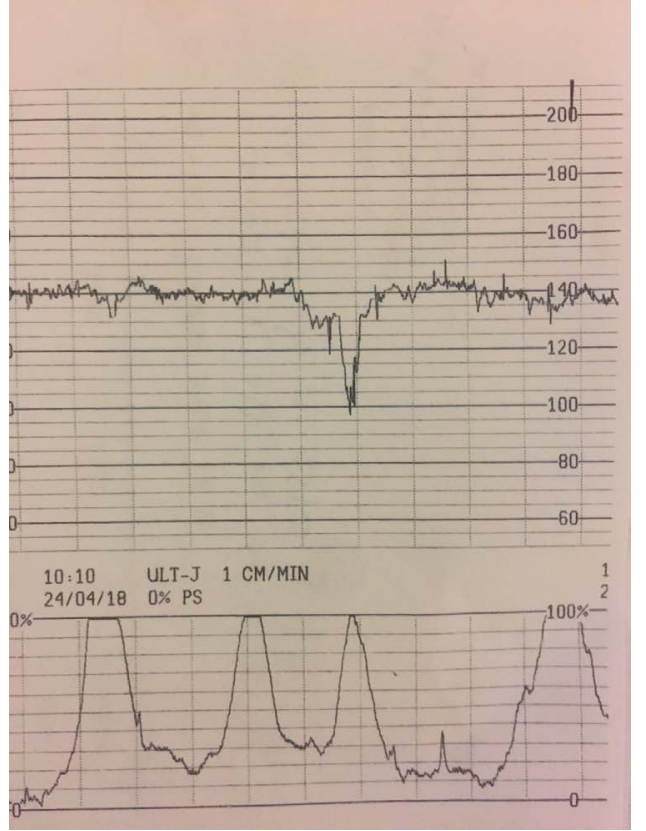

Figure 2. An early FHR trace deceleration the day of delivery by cesarean section

A cesarean section was immediately performed. A 2540 newborn in good conditions was delivered.

\section{Conclusion}

Spontaneous single decelerations in absence of uterine contractions, are infrequent situations. Most international reports describe single pregnancy cases. The etiology of this severe spontaneous deceleration in the absence of uterine contractions is unknown.
Intrauterine growth restriction and oligohydramnios were the more frequent complications in a previous report group (27\% and $20 \%$ respectively) [3]. Interestingly, we found that duration of decelerations was higher in high risk pregnancies, in agreement with other groups findings, which is possibly related to an hypoxic-ischemic derangement. Other groups have observed that absence of accelerations during labor is of little value in interpreting fetal heart rate patterns [4].

A deep isolated spontaneous deceleration may not be explained merely associated to complete or partial cord blood compression. In conclusion, once present, this FHR rate abnormality may represent fetal compromise and thus careful supervision. A single episode of spontaneous deceleration does not represent the indication for immediate cesarean section, independent of the gestational age. However, considering the high mortality reported by other groups in medical literature, our efforts for adequate management should include continuous FHR tracing and Doppler velocimetry study, once some alteration is observed (repeated decelerations, bradycardia, tachycardia, ARED, flow cephalization, etc.) a caesarean section should be performed.

\section{References}

1. Visser GH, Huisjes HJ (1977) Diagnostic value of the unstressed antepartum cardiotocogram. Br J Obstet Gynaecol. 84: 321-6. [Crossref]

2. Visser GH, Redman CW, Huisjes HJ, Turnbull AC (1980) Nonstressed antepartum heart rate monitoring: implications of decelerations after spontaneous contractions. $\mathrm{Am}$ J Obstet Gynecol. 138: 429-35. [Crossref]

3. Gioia S, Piazze JJ, Maranghi L, Anceschi M (2006) Isolated spontaneous fetal heart rate decelerations: prognostic significance. J Perinat Med. 34: 86-7. [Crossref]

4. Holzmann M, Wretler S, Nordström L (2016) Absence of accelerations during labor is of little value in interpreting fetal heart rate patterns. Acta Obstet Gynecol Scand. 95: 1097-103. [Crossref]

5. Roemer VM, Walden R (2012) Basic principles of the foetal heart rate during delivery without hypoxia and acidosis. Z Geburtshilfe Neonatol. 216: 11-21. [Crossref]

Copyright: (C2018 Piazze J. This is an open-access article distributed under the terms of the Creative Commons Attribution License, which permits unrestricted use, distribution, and reproduction in any medium, provided the original author and source are credited. 